\title{
On Cohen's proof of the Factorization Theorem
}

\author{
by JAN KISYŃSKI (Lublin) \\ Dedicated to the memory of Mieczystaw Altman
}

\begin{abstract}
Various proofs of the Factorization Theorem for representations of Banach algebras are compared with its original proof due to P. Cohen.

Various proofs of the Cohen-Hewitt Factorization Theorem for representations of Banach algebras are presented and discussed in [H-R;II], pp. 263290, [D-W], pp. 93-106 and 248-251, and [Pal], pp. 534-538.

The purpose of the present paper is to stress that all these proofs, and also some others, essentially rely upon a lemma which is implicitly contained in Cohen's paper [C]. This lemma appears below as Lemma 1 of Section 2.

Our discussion of various proofs of the Cohen-Hewitt Factorization Theorem is contained in Sections 2 and 3.2-3.4. Section 3.1 concerns the earlier factorization results of R. Salem, A. Zygmund and W. Rudin. In Sections 3.5-3.7 some papers are mentioned containing either more elaborate factorization theorems of the Cohen-Hewitt type or factorization results of another nature.
\end{abstract}

Acknowledgements. The author is greatly indebted to Wojciech Chojnacki for stimulating discussions and bibliographical hints.

1. The Cohen-Hewitt Factorization Theorem. The left approximate identity in a Banach algebra $A$ is, by definition, a net $\left(e_{\iota}\right)_{\iota \in I} \subset A$ such that

$$
\lim _{\iota}\left\|e_{\iota} a-a\right\|_{A}=0 \quad \text { for every } a \in A \text {. }
$$

2000 Mathematics Subject Classification: Primary 46H25; Secondary 43A20.

Key words and phrases: factorization for a representation of a Banach algebra with a bounded approximate identity. 
A left approximate identity $\left(e_{\iota}\right)_{\iota \in I} \subset A$ is said to be bounded if

$$
\sup _{\iota \in I}\left\|e_{\iota}\right\|_{A}<\infty \text {. }
$$

If $A$ is a Banach algebra with bounded left approximate identity $\left(e_{\iota}\right)_{\iota \in I}$ and $T$ is a continuous representation of $A$ on a Banach space $X$, then

$$
\lim _{\iota}\left\|T\left(e_{\iota}\right) y-y\right\|_{X}=0 \quad \text { for every } y \in \overline{\operatorname{span}} T(A) X .
$$

This follows from the fact that $\left(T\left(e_{\iota}\right)\right)_{\iota \in I}$ is a bounded net in $L(X)$ such that $\lim _{\iota}\left\|T\left(e_{\iota}\right) y-y\right\|=0$ for every $y \in T(A) X\left(^{1}\right)$.

The Factorization Theorem (P. Cohen [C], 1959; E. Hewitt [H], 1964). If $A$ is a Banach algebra with bounded left approximate identity $\left(e_{\iota}\right)_{\iota \in I}$ and $T$ is a continuous representation of $A$ on a Banach space $X$, then $T(A) X$ is a closed subspace of $X$. Furthermore, for every $y \in T(A) X$ and every $\varepsilon>0$ there are $a \in A$ and $x \in \overline{T(A) y}$ such that $T(a) x=y$, $\|x-y\| \leq \varepsilon$, and

$$
a=\sum_{n=1}^{\infty} p_{n} e_{\iota_{n}} \quad \text { where } \iota_{n} \in I, p_{n}>0 \text { for } n=1,2, \ldots \text { and } \sum_{n=1}^{\infty} p_{n}=1 .
$$

The assertions of the Factorization Theorem may be expressed by the single condition

(4) for every $y \in \overline{\operatorname{span}} T(A) X$ and every $\varepsilon>0$ there are $a \in A$ and $x \in \overline{T(A) y}$ such that $T(a) x=y,\|x-y\| \leq \varepsilon$ and (3) is satisfied.

2. Cohen's proof of the Factorization Theorem. Let $A$ be a Banach algebra, and $T$ a continuous representation of $A$ on a Banach space $X$. Suppose that there is a bounded left approximate identity in $A$. Then Cohen's proof of the existence of a factorization

$$
y=T(a) x, \quad a \in A, x \in X,
$$

of an element $y$ of $\overline{\operatorname{span}} T(A) X$ consists in approximation by a sequence of factorizations

$$
y=\widetilde{T}\left(a_{n}\right) x_{n}, \quad a_{n} \in G, x_{n}=\widetilde{T}\left(a_{n}^{-1}\right) y, n=1,2, \ldots,
$$

where $G$ is the set of invertible elements of the unitization $A_{\mathrm{u}}$ of $A$, and $\widetilde{T}$ is a representation of $A_{\mathrm{u}}$ on $X$ extending the representation $T$.

$\left({ }^{1}\right)$ From (2) it follows at once that $\overline{\operatorname{span}} T(A) X=\overline{T(A) X}$, i.e. $\overline{T(A) X}$ is a closed linear subspace of $X$. The factorization theorem says more: $T(A) X$ itself is a closed linear subspace. This last is almost trivial when $A$ contains a unit $e$. Indeed, since $T(e)=$ $T(e \cdot e)=T(e) T(e)$, it follows that $T(e)$ is a projector and hence $T(e) X$ is a closed subspace. Furthermore, $T(A) X=T(e A) X=T(e) T(A) X \subset T(e) X \subset T(A) X$, so that $T(A) X=T(e) X$. 
The unitization $A_{\mathrm{u}}$ of $A$ is the Banach algebra with unit such that

$1^{\circ}$ as a linear space, $A_{\mathrm{u}}$ is equal to the direct sum $\mathbb{K}+A$, where $\mathbb{K}$ is the field of scalars of $A$,

$2^{\circ} A_{\mathrm{u}}=\mathbb{K}+A$ is equipped with the norm \|\|$_{A_{\mathrm{u}}}$ such that $\|\lambda+a\|_{A_{\mathrm{u}}}=$ $|\lambda|+\|a\|_{A}$ for every $\lambda \in \mathbb{K}$ and $a \in A$,

$3^{\circ}$ the multiplication in $A_{\mathrm{u}}$ is defined by

$$
(\lambda+a)(\mu+b)=\lambda \mu+(\mu a+\lambda b+a b),
$$

where $\lambda, \mu \in \mathbb{K}$ and $a, b \in A$.

The unit in $A_{\mathrm{u}}$ is $1=1+0 \in \mathbb{K}+A\left(^{2}\right)$. Let

$$
\varphi: A_{\mathrm{u}} \rightarrow \mathbb{K}, \quad \pi: A_{\mathrm{u}} \rightarrow A
$$

be the projectors corresponding to the splitting $A_{\mathrm{u}}=\mathbb{K}+A$. From $3^{\circ}$ it follows that $\varphi$ is a multiplicative functional on $A_{\mathrm{u}}$. Let $\widetilde{T}$ be the representation of $A_{\mathrm{u}}$ on $X$ such that

$$
\widetilde{T}(a)=\varphi(a)+T(\pi a) \quad \text { for every } a \in A_{\mathrm{u}} .
$$

The following lemma is implicitly contained in Cohen's paper $[\mathrm{C}]$.

LEMmA 1. Under the assumptions of the Factorization Theorem, let

$$
\mathcal{E}=\left\{e_{\iota}: \iota \in I\right\}, \quad M=\sup _{e \in \mathcal{E}}\|e\|_{A} .
$$

Take any $y \in \overline{\operatorname{span}} T(A) X, a \in G, \gamma \in(0,1 /(M+1)), \delta>0$. Then there exists an $\widetilde{a} \in G$ satisfying the three conditions:

$$
\begin{aligned}
& \varphi(\widetilde{a})=\varphi(a)-\gamma \varphi(a), \\
& \pi \widetilde{a}=\pi a+\gamma \varphi(a) e \quad \text { for some } e \in \mathcal{E}, \\
& \left\|\widetilde{T}\left(\widetilde{a}^{-1}\right) y-\widetilde{T}\left(a^{-1}\right) y\right\|_{X} \leq \delta .
\end{aligned}
$$

Note that (i)\&(ii) may be written as one condition

$$
\widetilde{a}=a+\gamma \varphi(a)(e-1),
$$

and that (i)\&(ii) implies

$$
\|\pi \widetilde{a}-\pi a\|_{A} \leq \gamma M|\varphi(a)|=M|\varphi(\widetilde{a})-\varphi(a)| .
$$

Lemma 1 implies the Factorization Theorem. Let

$$
p_{n}=\lambda_{n-1}-\lambda_{n} \quad \text { for } n=1,2, \ldots,
$$

where $\lambda_{0}, \lambda_{1}, \ldots$ is a positive strictly decreasing sequence such that

$$
\begin{aligned}
& \lambda_{0}=1, \quad \lim _{n \rightarrow \infty} \lambda_{n}=0, \\
& 1-\frac{\lambda_{n}}{\lambda_{n-1}}=\gamma_{n} \in\left(0, \frac{1}{M+1}\right) \quad \text { for } n=1,2, \ldots
\end{aligned}
$$

$\left({ }^{2}\right)$ If $A$ has a unit $e$, then $A_{\mathrm{u}}$ makes sense, but $e$ is no longer a unit in $A_{\mathrm{u}}$. 
Then

$$
\begin{aligned}
& \lambda_{n}=\left(1-\gamma_{n}\right) \lambda_{n-1}, \\
& p_{n}=\gamma_{n} \lambda_{n-1}>0 \quad \text { for } n=1,2, \ldots, \quad \text { and } \quad \sum_{n=1}^{\infty} p_{n}=1 .
\end{aligned}
$$

The concrete form of the sequence $\lambda_{0}, \lambda_{1}, \ldots$ is inessential. One can follow $[\mathrm{C}]$ and take $\lambda_{n}=(1-\gamma)^{n}, \gamma=\mathrm{const} \in(0,1 /(M+1))$, so that $\gamma_{n}=\gamma$, or $\lambda_{n}=(1+M) /(1+M+n)$, so that $\gamma_{n}=1 /(1+M+n)$. From (5), (6) and (i) \&(v)\&(iii) it follows that for every $y \in \overline{\operatorname{span}} T(A) X$ and every $\varepsilon>0$ there exists a sequence $a_{0}, a_{1}, \ldots$ of elements of $G$ such that $a_{0}=1$ and

$$
\begin{aligned}
& \varphi\left(a_{n}\right)=\lambda_{n}, \\
& \left\|\pi a_{n}-\pi a_{n-1}\right\|_{A} \leq M p_{n}, \\
& \left\|\widetilde{T}\left(a_{n}^{-1}\right) y-\widetilde{T}\left(a_{n-1}^{-1}\right) y\right\|_{X} \leq \varepsilon p_{n}
\end{aligned}
$$

for $n=1,2, \ldots$ From $(\mathrm{i})_{0} \&(\mathrm{v})_{0} \&(\mathrm{iii})_{0}$ it follows that $\lim _{n \rightarrow \infty}\left\|a_{n}-a\right\|_{A_{\mathrm{u}}}=0$ for some $a \in A$ such that

$$
\|a\|_{A}=\left\|\pi \sum_{n=1}^{\infty}\left(a_{n}-a_{n-1}\right)\right\|_{A} \leq M \sum_{n=1}^{\infty} p_{n}=M
$$

and, if $x_{n}=\widetilde{T}\left(a_{n}^{-1}\right) y$, then $\lim _{n \rightarrow \infty}\left\|x_{n}-x\right\|_{X}=0$ for some $x \in X$ such that

$$
\|x-y\|_{X}=\left\|x-x_{0}\right\|_{X} \leq \sum_{n=1}^{\infty}\left\|x_{n}-x_{n-1}\right\|_{X} \leq \varepsilon \sum_{n=1}^{\infty} p_{n}=\varepsilon .
$$

Since $y=\widetilde{T}\left(a_{n}\right) x_{n}$ for every $n=0,1, \ldots$, a passage to the limit implies that $y=\widetilde{T}(a) x=T(a) x$. By $(2), y=\lim _{\iota} T\left(e_{\iota}\right) y \in \overline{T(A) y}$, whence

$$
x_{n}=\widetilde{T}\left(a_{n}^{-1}\right) y=\varphi\left(a_{n}^{-1}\right) y+T\left(\pi a_{n}^{-1}\right) y \in \overline{T(A) y}
$$

for $n=0,1, \ldots$, and so $x=\lim _{n \rightarrow \infty} x_{n} \in \overline{T(A) y}$. Thus (i)\&(v)\&(iii) implies $(4)_{M} \quad$ for every $y \in \overline{\operatorname{span}} T(A) X$ and every $\varepsilon>0$ there are $a \in A$ and $x \in \overline{T(A) y}$ such that $\|a\|_{A} \leq M,\|x-y\|_{X} \leq \varepsilon$ and $T(a) x=y$.

The statement $(4)_{M}$ is a weakened version of (4): the condition (3) is replaced by $a \in A,\|a\|_{A} \leq M$. In order to prove (4), instead of (i)\&(v)\&(iii) one has to use (iv)\&(iii). Indeed, (iv)\&(iii) implies that for every $y \in \overline{\operatorname{span}} T(A) X$ and every $\varepsilon>0$ there exists a sequence $a_{0}, a_{1}, \ldots$ of elements of $G$ such that $a_{0}=1$, the conditions $(\mathrm{i})_{0}$ and (iii) $)_{0}$ are satisfied, and

(iv) $0_{0} \quad$ for every $n=1,2, \ldots$ there is $e_{n} \in \mathcal{E}$ such that

$$
a_{n}=a_{n-1}+\gamma_{n} \varphi\left(a_{n-1}\right)\left(e_{n}-1\right)=a_{n-1}+p_{n}\left(e_{n}-1\right) .
$$

Since (iv) $)_{0}$ implies $(\mathrm{v})_{0}$, the preceding argument leading to $(4)_{M}$ remains in 
force. Furthermore, from (iv) 0 it follows that $a_{n}=\lambda_{n}+p_{1} e_{1}+\ldots+p_{n} e_{n}$ for every $n=1,2, \ldots$ and hence, by (5) and (6), $\lim _{n \rightarrow \infty}\left\|a_{n}-a\right\|_{A_{\mathrm{u}}}=0$, where $a=\sum_{n=1}^{\infty} p_{n} e_{n}$.

Proof of Lemma $1\left(^{3}\right)$. For every $\iota \in I$ define

$$
\widetilde{a}_{\iota}=a+b_{\iota}, \quad b_{\iota}=\gamma \varphi(a)\left(e_{\iota}-1\right) .
$$

Lemma 1 will follow once it is shown that

$$
\text { there is } \iota_{0} \in I \text { such that } \widetilde{a}_{\iota} \in G \text { whenever } \iota_{0} \prec \iota \text {, }
$$

and

$$
\lim _{\iota}\left\|\widetilde{T}\left(\widetilde{a}_{\iota}^{-1}\right) y-\widetilde{T}\left(a^{-1}\right) y\right\|_{X}=0 .
$$

In the proof of (I) we will use the equality

$$
\widetilde{a}_{\iota}=\left(1+b_{\iota} a^{-1}\right) a .
$$

Notice that

$$
\begin{aligned}
b_{\iota} a^{-1} & =\gamma \varphi(a)\left(e_{\iota}-1\right)\left[\varphi\left(a^{-1}\right)+\pi a^{-1}\right] \\
& =\gamma\left(e_{\iota}-1\right)+\gamma \varphi(a)\left(e_{\iota}-1\right) \pi a^{-1}
\end{aligned}
$$

whence

$$
\left\|b_{\iota} a^{-1}\right\|_{A_{\mathrm{u}}} \leq \gamma(M+1)+\gamma|\varphi(a)| \cdot\left\|\left(e_{\iota}-1\right) \pi a^{-1}\right\|_{A} .
$$

Since $\gamma \in(0,1 /(M+1))$, one can choose $\theta$ such that

$$
\gamma(M+1)<\theta<1 \text {. }
$$

By (1) and (7), there is $\iota_{0} \in I$ such that

$$
\left\|b_{\iota} a^{-1}\right\|_{A_{\mathrm{u}}} \leq \theta \quad \text { whenever } \iota_{0} \prec \iota .
$$

An application of the C. Neumann series shows that then $1+b_{\iota} a^{-1} \in G$ and $\left\|\left(1+b_{\iota} a^{-1}\right)^{-1}\right\|_{A_{u}} \leq(1-\theta)^{-1}$. In consequence, whenever $\iota_{0} \prec \iota$, then $\widetilde{a}_{\iota}=\left(1+b_{\iota} a^{-1}\right) a \in G\left({ }^{4}\right)$ and

$$
\left\|\widetilde{a}_{\iota}^{-1}\right\|_{A_{\mathrm{u}}} \leq\left\|a^{-1}\right\|_{A_{\mathrm{u}}}(1-\theta)^{-1} .
$$

For the proof of (II) observe that if $\iota_{0} \prec \iota$, then

$$
\widetilde{a}_{\iota}^{-1}-a^{-1}=\widetilde{a}_{\iota}^{-1}\left(a-\widetilde{a}_{\iota}\right) a^{-1}=-\widetilde{a}_{\iota}^{-1} b_{\iota} a^{-1}=-\gamma \varphi(a) \widetilde{a}_{\iota}^{-1}\left(e_{\iota}-1\right) a^{-1} .
$$

As a consequence, by (8),

$$
\begin{aligned}
& \left\|\widetilde{T}\left(\widetilde{a}_{\iota}^{-1}\right) y-\widetilde{T}\left(a^{-1}\right) y\right\|_{X} \\
& \quad \leq \gamma|\varphi(a)| \cdot\|\widetilde{T}\|_{L\left(A_{\mathrm{u}} ; L(X)\right)}\left\|a^{-1}\right\|_{A_{\mathrm{u}}}(1-\theta)^{-1}\left\|\left[T\left(e_{\iota}\right)-1\right] \widetilde{T}\left(a^{-1}\right) y\right\|_{X} .
\end{aligned}
$$

$\left({ }^{3}\right)$ This proof differs from the argument in [C]; see Section 3.2.

$\left({ }^{4}\right)$ This proof of the invertibility of $\widetilde{a}_{\iota}$ follows [P;3], pp. 283-284, and [P-P], p. 136. The elements $b, b^{\prime}$ and $c$ of $A_{\mathrm{u}}$ appearing in [P;3] correspond to our $a^{-1}, \widetilde{a}_{\iota}^{-1}$ and $(1+$ $\left.b_{\iota} a^{-1}\right)^{-1}$. In $[\mathrm{P}-\mathrm{P}]$ the elements of $A_{\mathrm{u}}$ are written in the form $a+r, a \in A, r \in \mathbb{K}$, so that our $\pi a^{-1}$ corresponds to $(a+r)^{-1}-r^{-1}$ in $[\mathrm{P}-\mathrm{P}]$. 
From this estimate and from (2) the equality (II) follows, because $\widetilde{T}\left(a^{-1}\right) y=$ $\varphi\left(a^{-1}\right) y+T\left(\pi a^{-1}\right) y \in \overline{\operatorname{span}} T(A) X$.

3. Forerunners of the Cohen-Hewitt Factorization Theorem, variants of the proof, and references to other factorization results

3.1. Forerunners of the Cohen-Hewitt theorem. Let $G$ be a locally compact group with a fixed left-invariant Haar measure $\mu$, and let $L^{1}(G)$ be the space of the equivalence classes of functions on $G$ integrable with respect to $\mu$. The convolution $a * b$ of two elements, $a$ and $b$, of $L^{1}(G)$ is defined by

$$
(a * b)(g)=\int_{G} a(h) b\left(h^{-1} g\right) \mu(d h) \quad \text { for a.e. } g \in G .
$$

Then $L^{1}(G)$ is a convolution Banach algebra. Cohen's paper [C] contains the following results which follow from the Factorization Theorem applied to $A=L^{1}(G), X=L^{1}(G)$, or $X=C(G)$ if $G$ is compact, and to the representation of $A$ on $X$ defined by $T(a) x=a * x$ :

(a) $L^{1}(G)=L^{1}(G) * L^{1}(G)$ for every locally compact group $G$. This was proved earlier in particular cases: for $G=\mathbb{T}$, the circle group, by R. Salem and A. Zygmund, and for $G=\mathbb{R}$ and $G$ a locally euclidian abelian group by W. Rudin [R;1], [R;2].

(b) $C(G)=L^{1}(G) * C(G)$ for every compact group $G$. In the particular case of $G=\mathbb{T}$ this was proved earlier by R. Salem and A. Zygmund.

(c) Strictly positive factorization: if $G$ is a compact group, $y \in C(G)$ and $\inf _{G} y>0$, then there are $a \in L^{1}(G)$ and $x \in C(G)$ such that ess $\inf _{G} a>0$, $\inf _{G} x>0$ and $a * x=y$. In the deduction of this result from the Factorization Theorem the conditions (3) and $\|x-y\| \leq \varepsilon$ are essential. Note that Cohen $[\mathrm{C}]$ proved that an analogous non-negative factorization does not hold.

In their proofs of the particular cases of (a) and (b), R. Salem, A. Zygmund and W. Rudin used the Fourier methods which are not applicable in the general situation. Nevertheless it seems interesting to compare the condition (3) from the factorization theorem with the formulas labelled below by $(*)$ and $\left(*_{*}^{*}\right)$, appearing in the proof of the factorization $L^{1}(\mathbb{R})=$ $L^{1}(\mathbb{R}) * L^{1}(\mathbb{R})$ presented in $[\mathrm{R} ; 2]$. The approximate identity in $L^{1}(\mathbb{R})$ appears there in the from $e_{t}=K_{t}$ for $t \in(0, \infty)$, where

$$
K_{t}(x)=\frac{t}{2 \pi}\left(\frac{\sin (t x / 2)}{t x / 2}\right)^{2}
$$

is the Fejér kernel. The Fourier transform of $K_{t}$ is

$$
\widehat{K}_{t}(\xi)=(1-|\xi| / t)^{+} .
$$


For given $f \in L^{1}(\mathbb{R})$ Rudin proves that $f=a * g$ where $a \in L^{1}(\mathbb{R})$ and $g \in L^{1}(\mathbb{R})$ are defined as the integrals of continuous $L^{1}(\mathbb{R})$-valued functions

$$
\begin{aligned}
& a=\int_{0}^{\infty} t \lambda^{\prime \prime}(t) K_{t} d t, \\
& g=f+\int_{0}^{\infty} \alpha(t)\left(f-K_{t} * f\right) d t .
\end{aligned}
$$

Here $\alpha \in C[0, \infty)$ and $\lambda \in C^{2}[0, \infty)$ are positive functions such that

$$
\int_{0}^{\infty} \alpha(t) d t=\infty, \quad \int_{0}^{\infty} \alpha(t)\left(\left\|K_{t} * f-f\right\|+\frac{1}{t}\right) d t<\infty, \quad \lambda(t)=\frac{1}{\Phi(t)},
$$

where

$$
\Phi(t)=1+\int_{0}^{t} \alpha(s) d s+t \int_{t}^{\infty} \frac{\alpha(s)}{s} d s .
$$

These definitions imply that $g \in L^{1}(\mathbb{R}), \lambda^{\prime \prime}$ is positive and

$$
\int_{0}^{\infty} t \lambda^{\prime \prime}(t) d t=1
$$

so that $a \in L^{1}(\mathbb{R})$, and furthermore, $\widehat{a}(\xi)=\lambda(|\xi|)$ and $\widehat{g}(\xi)=\Phi(|\xi|) \widehat{f}(\xi)\left({ }^{5}\right)$, whence $\widehat{a} \widehat{g}=\widehat{f}$, and so $a * g=f$.

Discrete forerunners of $(*)$ and $(* *)$ appear in the book of A. Zygmund [Z;I] as (1.7) on p. 183, (4.2) on p. 93, and Theorem 1.5 on p. 183 (attributed on p. 378 to H. W. Young (1913) and A. N. Kolmogorov (1923)). A discrete forerunner of $\left(\begin{array}{l}* \\ *\end{array}\right)$ is the second formula in line $111^{3}$ of R. Salem's paper [S], related to a limit passage in (3) on p. 110 of [S]. A. Zygmund [Z;I], p. 378 ${ }_{11-6}$, points out that the theorems from $[\mathrm{S}]$ together with Theorem 1.5 of Chap. V of $[\mathrm{Z} ; \mathrm{I}]$, p. 183, lead to the factorization results for $G=\mathbb{T}$ mentioned in (a) and (b). A discrete analogue of the whole elegant Rudin construction is used in Sec. 7.5 of the book of R. E. Edwards [E;I] for reproving the SalemZygmund results. Remarks on the Fourier factorization may be found in Sec. 3.1.1(c) and 7.5.2-4 of [E;I] and Sec. 11.4.18(6) of [E;II].

$\left({ }^{5}\right)$ Indeed, $\Phi^{\prime}(t)=\int_{t}^{\infty}(\alpha(s) / s) d s, \Phi^{\prime \prime}(t)=-\alpha(t) / t \leq 0, \lambda^{\prime}(t)=-\Phi^{\prime}(t) /[\Phi(t)]^{2} \leq 0$, $\lambda^{\prime \prime}(t)=\left(2\left[\Phi^{\prime}(t)\right]^{2}-\Phi^{\prime \prime}(t) \Phi(t)\right)[\Phi(t)]^{-3} \geq 0$. Hence $0 \leq-t \lambda^{\prime}(t)=t \Phi^{\prime}(t) /[\Phi(t)]^{2} \leq$ $[\Phi(t)]^{-1}$, so $\lim _{t \rightarrow \infty} t \lambda^{\prime}(t)=\lim _{t \rightarrow \infty} \lambda(t)=0$ and $\int_{0}^{\infty} t \lambda^{\prime \prime}(t) d t=\left.t \lambda^{\prime}(t)\right|_{t=0} ^{t=\infty}-\int_{0}^{\infty} \lambda^{\prime}(t) d t$ $=\lambda(0)=1$. Moreover, $(*)$ implies $\widehat{a}(\xi)=\int_{0}^{\infty} t \lambda^{\prime \prime}(t)(1-|\xi| / t)^{+} d t=\int_{|\xi|}^{\infty}(t-|\xi|) \lambda^{\prime \prime}(t) d t=$ $\left.(t-|\xi|) \lambda^{\prime}(t)\right|_{t=|\xi|} ^{t=\infty}-\int_{|\xi|}^{\infty} \lambda^{\prime}(t) d t=\lambda(|\xi|)$, and $\widehat{g}(\xi)=\left[1+\int_{0}^{\infty} \alpha(t)\left(1-\widehat{K}_{t}(\xi)\right) d t\right] \widehat{f}(\xi)=$ $\Phi(|\xi|) \widehat{f}(|\xi|)$ by $\left(\begin{array}{c}* \\ *\end{array}\right)$. 
3.2. The original calculations of Cohen. As already mentioned, Lemma 1 is implicitly contained in Cohen's paper $[\mathrm{C}]$. The argument used in $[\mathrm{C}]$ for proving this implicit lemma was repeated in $[\mathrm{V}],[\mathrm{H}],[\mathrm{C}-\mathrm{FT}],[\mathrm{J}],[\mathrm{G}-\mathrm{L}-\mathrm{R}]$, $[\mathrm{T}],[\mathrm{S}-\mathrm{T}],[\mathrm{H}-\mathrm{R} ; \mathrm{II}],[\mathrm{B}-\mathrm{D}],[\mathrm{A}-\mathrm{S}],[\mathrm{D}-\mathrm{W}],[\mathrm{G}]$ and $[\mathrm{Pal}]$. In our notation, this argument is as follows. For every $\iota \in I$ one has $\left\|\gamma\left(e_{\iota}-1\right)\right\| \leq \gamma(M+1)=$ $\vartheta<1$, whence $1-\gamma+\gamma e_{\iota} \in G$ and $\left\|\left(1-\gamma+\gamma e_{\iota}\right)^{-1}\right\| \leq(1-\vartheta)^{-1}$. The possibility of choosing $\iota_{0} \in I$ so that

$$
\widetilde{a}_{\iota}=a+\gamma \varphi(a)\left(e_{\iota}-1\right) \in G \quad \text { whenever } \iota_{0} \prec \iota
$$

is proved in $[\mathrm{C}]$ by means of the formulas

$$
\widetilde{a}_{\iota}=\left(1-\gamma+\gamma e_{\iota}\right) a_{\iota}, \quad a_{\iota}=\varphi(a)+\left(1-\gamma+\gamma e_{\iota}\right)^{-1} \pi a .
$$

Since

$$
\begin{aligned}
\left\|a_{\iota}-a\right\| & =\left\|\left(1-\gamma+\gamma e_{\iota}\right)^{-1} \pi a-\pi a\right\| \\
& =\left\|\gamma\left(1-\gamma+\gamma e_{\iota}\right)^{-1}\left(1-e_{\iota}\right) \pi a\right\| \\
& \leq \gamma(1-\vartheta)^{-1}\left\|e_{\iota} \pi a-\pi a\right\|,
\end{aligned}
$$

it follows by (1) that

$$
\lim _{\iota}\left\|a_{\iota}-a\right\|=0 .
$$

Hence there is $\iota_{0} \in I$ such that if $\iota_{0} \prec \iota$, then $\left\|a_{\iota}-a\right\| \leq \frac{1}{2}\left\|a^{-1}\right\|^{-1}$, and so $a_{\iota}=\left[1+\left(a_{\iota}-a\right) a^{-1}\right] a \in G$ and $\left\|a_{\iota}^{-1}\right\| \leq 2\left\|a^{-1}\right\|$. In consequence, if $\iota_{0} \prec \iota$, then $\widetilde{a}_{\iota}=\left(1-\gamma+\gamma e_{\iota}\right) a_{\iota} \in G$, proving (I). The equality

$$
\lim _{\iota}\left\|\widetilde{T}\left(\widetilde{a}_{\iota}^{-1}\right) y-\widetilde{T}\left(a^{-1}\right) y\right\|=0
$$

is proved in $[\mathrm{C}]$ as follows. If $\iota_{0} \prec \iota$, then

$$
\begin{aligned}
\widetilde{a}_{\iota}^{-1}-a^{-1}= & \left(a_{\iota}^{-1}-a^{-1}\right)\left(1-\gamma+\gamma e_{\iota}\right)^{-1}+a^{-1}\left[\left(1-\gamma+\gamma e_{\iota}\right)^{-1}-1\right] \\
= & -a_{\iota}^{-1}\left(a_{\iota}-a\right) a^{-1}\left(1-\gamma+\gamma e_{\iota}\right)^{-1} \\
& -\gamma a^{-1}\left(1-\gamma+\gamma e_{\iota}\right)^{-1}\left(e_{\iota}-1\right),
\end{aligned}
$$

whence

$$
\begin{aligned}
& \left\|\widetilde{T}\left(\widetilde{a}_{\iota}^{-1}\right) y-\widetilde{T}\left(a^{-1}\right) y\right\| \\
& \quad \leq\|\widetilde{T}\| \cdot\left\|a^{-1}\right\|(1-\vartheta)^{-1}\left\{2\left\|a^{-1}\right\| \cdot\|y\| \cdot\left\|a_{\iota}-a\right\|+\gamma\left\|T\left(e_{\iota}\right) y-y\right\|\right\} .
\end{aligned}
$$

This estimate implies (II), by (10) and (2).

3.3. Variants of Cohen's proof. The method of Koosis. The reasoning presented in Section 3.2 becomes simpler when the elements $\widetilde{a}_{\iota}=a+$ $\gamma \varphi(a)\left(e_{\iota}-1\right)$ satisfying $(9)$ are replaced by

$$
\widehat{a}_{\iota}=\left(1-\gamma+\gamma e_{\iota}\right) a .
$$

Then, for every $\iota \in I, \varphi\left(\widehat{a}_{\iota}\right)=(1-\gamma) \varphi(a)$, 


$$
\left\|\pi \widehat{a}_{\iota}-\pi a\right\|=\left\|\gamma \varphi(a) e_{\iota}+\gamma\left(e_{\iota}-1\right) \pi a\right\| \leq \gamma|\varphi(a)| M+\gamma\left\|e_{\iota} \pi a-\pi a\right\|,
$$

$\widehat{a}_{\iota} \in G$, and $\widehat{a}_{\iota}^{-1}-a^{-1}=-\gamma a^{-1}\left(1-\gamma+\gamma e_{\iota}\right)^{-1}\left(e_{\iota}-1\right)$, whence

$\left\|\widetilde{T}\left(\widehat{a}_{\iota}^{-1}\right) y-\widetilde{T}\left(a^{-1}\right) y\right\| \leq \gamma\|\widetilde{T}\| \cdot\left\|a^{-1}\right\|(1-\vartheta)^{-1}\left\|T\left(e_{\iota}\right) y-y\right\|, \quad \vartheta=\gamma(M+1)$.

This yields

LEmma 2. Under the notation of Sections 1 and 2, and the assumptions of the Factorization Theorem, let $y \in \overline{\operatorname{span}} T(A) X, a \in G, \gamma \in$ $(0,1 /(M+1)), \delta>0$. Then there is $\widehat{a} \in G$ such that $\widehat{a}=(1-\gamma+\gamma e) a$ for some e $\in \mathcal{E}$,

$$
\|\pi \widehat{a}-\pi a\| \leq \gamma|\varphi(a)| M+\delta \quad \text { and } \quad\left\|\widetilde{T}\left(\widehat{a}^{-1}\right) y-\widetilde{T}\left(a^{-1}\right) y\right\| \leq \delta .
$$

For given $y \in \overline{\operatorname{span}} T(A) X, \varepsilon>0$ and $\gamma \in(0,1 /(M+1))$, using Lemma 2, one can define inductively $e_{1}, e_{2}, \ldots$ in $\mathcal{E}$ so that the formulas

$$
a_{0}=1, \quad a_{n}=\left(1-\gamma+\gamma e_{n}\right) a_{n-1} \quad \text { for } n=1,2, \ldots
$$

define a sequence $a_{0}, a_{1}, \ldots$ in $G$ such that $\varphi\left(a_{n}\right)=(1-\gamma)^{n},\left\|\pi a_{n}-\pi a_{n-1}\right\| \leq$ $\gamma(1-\gamma)^{n-1}(M+\varepsilon)$ and $\left\|\widetilde{T}\left(a_{n}^{-1}\right) y-\widetilde{T}\left(a_{n-1}^{-1}\right) y\right\| \leq \gamma(1-\gamma)^{n-1} \varepsilon$ for $n=1,2, \ldots$ This yields

$(\widehat{4})_{M} \quad$ for every $y \in \overline{\operatorname{span}} T(A) X$ and every $\varepsilon>0$ there are $\widehat{a} \in A$ and $\widehat{x} \in \overline{T(A) y}$ such that $\|\widehat{a}\| \leq M+\varepsilon,\|\widehat{x}-y\| \leq \varepsilon$ and $T(\widehat{a}) \widehat{x}=y$.

If, for given $y \in \overline{\operatorname{span}} T(A) X$ and $\varepsilon>0, \widehat{a}$ and $\widehat{x}$ satisfy $(\widehat{4})_{M}$, then for $a=\frac{M}{M+\varepsilon} \widehat{a}$ and $x=\frac{M+\varepsilon}{M} \widehat{x}$ one has $\|a\| \leq M$ and

$\|x-y\| \leq\|\widehat{x}-y\|+\frac{\varepsilon}{M}\|\widehat{x}\| \leq\|\widehat{x}-y\|+\frac{\varepsilon}{M}\|y\|+\frac{\varepsilon}{M}\|\widehat{x}-y\| \leq\left(1+\frac{\|y\|+\varepsilon}{M}\right) \varepsilon$.

Hence $(\widehat{4})_{M}$ is equivalent to $(4)_{M}$ from Section 2 .

It was $\mathrm{P}$. Koosis $[\mathrm{K}]$ who showed that the elements $a \in A$ and $x \in$ $\overline{T(A) y}$ satisfying $T(a) x=y$ for a given $y \in \overline{\operatorname{span}} T(A) X$ may be determined by an approximation $a=\lim a_{n}, x=\lim \widetilde{T}\left(a_{n}^{-1}\right) y$, where $a_{0}=1 \in A_{\mathrm{u}}$, $a_{n}=\left(1-\gamma+\gamma e_{n}\right) a_{n-1}$ for $n=1,2, \ldots, \gamma=$ const $\in(0,1 /(M+1))$, and $e_{1}, e_{2}, \ldots$ is a suitable sequence of elements of $\mathcal{E}$. This idea was continued by M. Altman $[\mathrm{A} ; 1-5]$, R. S. Doran and J. Wichmann [D-W], pp. 97-100, J. Esterle [Es;4] and H. G. Feichtinger and M. Leinert [F-L], still for Banach algebras.

W. Żelazko [Ż], Sec. 6.4, pp. 24-26, showed that the Koosis-Altman formulas (11) may be replaced by

$$
a_{0}=1, \quad a_{n}=\Phi_{\gamma}\left(e_{n}\right) a_{n-1} \quad \text { for } n=1,2, \ldots,
$$

where $\gamma>0$ is a constant such that $\gamma(M-1)<1$, and

$$
\Phi_{\gamma}(e)=(1+\gamma-\gamma e)^{-1}
$$


for every $e \in \mathcal{E}$. Then

$$
\begin{gathered}
\left\|\Phi_{\gamma}(e)\right\| \leq(1+\gamma-\gamma M)^{-1}, \quad \Phi_{\gamma}(e)-1=\gamma \Phi_{\gamma}(e)(e-1), \\
{\left[\Phi_{\gamma}(e)\right]^{-1}-1=-\gamma(e-1),}
\end{gathered}
$$

which implies

LEMma 3. Under the notation of Sections 1 and 2, and the assumptions of the Factorization Theorem, let $y \in \overline{\operatorname{span}} T(A) X, a \in G, \gamma>0$, $\gamma(M-1)<1, \delta>0$. Then there is $e \in \mathcal{E}$ such that if $\widehat{\hat{a}}=\Phi_{\gamma}(e) a$, then $\widehat{\widehat{a}} \in G$,

$\|\pi \widehat{\widehat{a}}-\pi a\|=\|\widehat{\widehat{a}}-a\|-|\varphi(\widehat{\widehat{a}})-\varphi(a)| \leq \frac{\gamma(1+M)}{1+\gamma-\gamma M}|\varphi(a)|+\delta-\frac{\gamma}{1+\gamma}|\varphi(a)|$

and

$$
\left\|\widetilde{T}\left(\widehat{a}^{-1}\right) y-\widetilde{T}(a) y\right\| \leq \delta .
$$

For given $y \in \overline{\operatorname{span}} T(A) X, \varepsilon>0$ and $\gamma>0$ such that $\gamma(M-1)<1$, using Lemma 3 , one can define inductively $e_{1}, e_{2}, \ldots$ in $\mathcal{E}$ so that the sequence $a_{0}, a_{1}, \ldots$ of elements of $G$ defined by (12) satisfies

$$
\begin{gathered}
\varphi\left(a_{n}\right)=(1+\gamma)^{-n}, \\
\left\|\pi a_{n}-\pi a_{n-1}\right\| \leq\left(\frac{\gamma(1+M)}{1+\gamma-\gamma M}-\frac{\gamma}{1+\gamma}\right)(1+\gamma)^{1-n}+\frac{\varepsilon}{2^{n+1}},
\end{gathered}
$$

and $\left\|\widetilde{T}\left(a_{n}^{-1}\right) y-\widetilde{T}\left(a_{n-1}^{-1}\right) y\right\| \leq \varepsilon / 2^{n}$. If $a=\lim a_{n}$ and $x=\lim \widetilde{T}\left(a_{n}^{-1}\right) y$, then $a \in A, x \in \overline{T(A) y}, y=T(a) x,\|x-y\| \leq \varepsilon$ and

$$
\|a\| \leq \sum_{n=1}^{\infty}\left\|\pi a_{n}-\pi a_{n-1}\right\| \leq \frac{(1+\gamma)(1+M)}{1+\gamma-\gamma M}-1+\frac{\varepsilon}{2} .
$$

For $\gamma>0$ such that

$$
\gamma(M-1)<1 \text { and } \frac{(1+\gamma)(1+M)}{1+\gamma-\gamma M} \leq 1+M+\frac{\varepsilon}{2},
$$

one obtains $(\widehat{4})_{M}$, which is equivalent to $(4)_{M}$.

In $[\mathrm{H}-\mathrm{R} ; \mathrm{II}]$, Sec. 32.50, pp. 287-288, an argument similar to that of Żelazko is carried out under the assumptions that $M=1$ and the approximate identity is two-sided. Instead of (12) Hewitt and Ross use the formula $a_{n}=a_{n-1} \varphi\left(e_{n}\right)$ where $\varphi(e)=\frac{1}{3}\left[1+\sum_{k=1}^{\infty}\left(\frac{1}{3}[1+e]\right)^{k}\right]$ is equal to our $\Phi_{1}(e)=(2-e)^{-1}=\frac{1}{3}\left(1-\frac{1}{3}[1+e]\right)^{-1}$. A construction similar to that of Żelazko also appears in [Es;1] in the proof of Theorem 2.8.

In Section 3.5 some papers will be mentioned in which the constructions of Koosis and Żelazko are applied to representations of some non-Banach algebras. These applications are possible thanks to the fact that these constructions lead to sequences of factorizations $y=\widetilde{T}\left(a_{n}\right) x_{n}, x_{n}=\widetilde{T}\left(a_{n}^{-1}\right) y$, 
for which the proofs of the invertibility of $a_{n}$ 's and of the existence of $\lim \widetilde{T}\left(a_{n}^{-1}\right) y$ are simpler than in the case of Cohen's construction.

3.4. Deduction of the Cohen-Hewitt Factorization Theorem from some results of $V$. Pták and J. Esterle. In a somewhat weakened version, namely with the condition $a \in \overline{\operatorname{conv}}\left\{e_{\iota}: \iota \in I\right\}$ but without $(3)\left({ }^{6}\right)$, the Factorization Theorem may be deduced from the "induction theorem" of V. Pták $([\mathrm{P} ; 3]$, p. $280 ;[\mathrm{P}-\mathrm{P}]$, p. $5 ;[\mathrm{P} ; 1] ;[\mathrm{P} ; 2])$, and also from the theorem of J. Esterle about projective systems ([Es;4], p. 109, Th. 2.1). The applicability of these theorems to the factorization problem follows from Lemmas 1-3. The proofs of both theorems are similar to the part of Cohen's argument presented above as "Lemma 1 implies the Factorization Theorem". Nevertheless, the approach to factorization via the theorem of Pták or that of Esterle is interesting because it emphasizes the geometric nature of Cohen's construction.

Under the notation of Sections 1 and 2, consider in $A_{\mathrm{u}}$ the subset

$$
E=\operatorname{conv}(\{1\} \cup \mathcal{E})=\bigcup_{0 \leq \lambda \leq 1}(\lambda+(1-\lambda) \operatorname{conv} \mathcal{E})
$$

Take any $y \in \overline{\operatorname{span}} T(A) X$ and $\varepsilon>0$. For every $\lambda \in(0,1]$ let

$$
\begin{aligned}
Z(\lambda)=\{(a, x) \in & (E \cap G) \times X: \\
& \|x-y\| \leq \varepsilon(1-\varphi(a)), \widetilde{T}(a) x=y, 0<\varphi(a) \leq \lambda\} .
\end{aligned}
$$

Then $(1, y) \in Z(1)$ and $Z\left(\lambda^{\prime}\right) \subset Z\left(\lambda^{\prime \prime}\right) \subset(E \cap G) \times \overline{T(A) y}$ whenever $0<$ $\lambda^{\prime}<\lambda^{\prime \prime} \leq 1$. If $(a, x) \in \bigcap_{0<\lambda \leq 1} \overline{Z(\lambda)}$, then $a \in \overline{\operatorname{conv}} \mathcal{E} \subset A, x \in \overline{T(A) y}$, $\|x-y\| \leq \varepsilon$, and $T(a) x=y$. Hence the weakened version of the statement (4) will follow once it is shown that

$$
\bigcap_{0<\lambda \leq 1} \overline{Z(\lambda)} \neq \emptyset \text {. }
$$

A proof of $(*)$ may be obtained by using Lemma 1 and applying Pták's or Esterle's theorem. Indeed, Lemma 1 implies

Corollary. Fix any $y \in \overline{\operatorname{span}} T(A) X$ and $\varepsilon>0$. Then for every $\lambda \in$ $(0,1],(a, x) \in Z(\lambda)$ and $\gamma \in(0,1 /(M+1))$ there exists $(\widetilde{a}, \widetilde{x}) \in Z((1-\gamma) \lambda)$ such that $\varphi(\widetilde{a})=(1-\gamma) \varphi(a)$ and

$$
\frac{\|\pi \widetilde{a}-\pi a\|}{M} \vee \frac{\|\widetilde{x}-x\|}{\varepsilon} \leq \gamma \varphi(a)=\varphi(a)-\varphi(\widetilde{a}) .
$$

Define in $A_{\mathrm{u}} \times X$ the norm

$$
\|(a, x)\|=\left(\frac{\|\pi a\|}{M} \vee \frac{\|x\|}{\varepsilon}\right)+|\varphi(a)|, \quad a \in A_{\mathrm{u}}, x \in X .
$$

$\left({ }^{6}\right)$ Sometimes this may be an essential loss of information, see (c) in Section 3.1. 
From the Corollary it follows that for every $\lambda \in(0,1],(a, x) \in Z(\lambda)$ and $\gamma \in(0,1 /(M+1))$ there is $(\widetilde{a}, \widetilde{x}) \in Z((1-\gamma) \lambda)$ such that $\|(\widetilde{a}, \widetilde{x})-(a, x)\| \leq$ $2 \gamma \lambda$. Since $(1, y) \in Z(1)$, it follows that $Z(\lambda) \neq \emptyset$ for every $\lambda \in(0,1]$, and

$$
\sup _{(a, x) \in Z(\lambda)} \operatorname{dist}((a, x) ; Z((1-\gamma) \lambda)) \leq 2 \gamma \lambda
$$

for every $\lambda \in(0,1]$ and $\gamma \in(0,1 /(M+1))$, where the distance is defined by the norm \|\|$\|$.

The implication $\left(\begin{array}{c}* \\ *\end{array}\right) \Rightarrow(*)$ is a consequence of Pták's theorem, and also of Esterle's. Indeed, if $\widetilde{Z}(t)=Z(t /(2 \gamma))$ for $t \in(0,2 \gamma]$, then $\left(\begin{array}{l}* \\ *\end{array}\right)$ takes the form

$$
\sup _{(a, x) \in \widetilde{Z}(t)} \operatorname{dist}((a, x) ; \widetilde{Z}(w(t))) \leq t \quad \text { for every } t \in(0,2 \gamma],
$$

where $w(t)=(1-\gamma) t$. Since $w(t)+(w \circ w)(t)+\ldots=\sum_{n=1}^{\infty}(1-\gamma)^{n} t<\infty$ for every $t \in(0,2 \gamma]$, the function $w$ is a rate of convergence on $(0,2 \gamma]([\mathrm{P}-\mathrm{P}]$, p. 2, Def. 1). Hence the theorem of Pták ([P-P], p. 5, Proposition 1.7) implies that $\bigcap_{0<t \leq 2 \gamma} \overline{\widetilde{Z}(t)} \neq \emptyset$, proving $(*)$.

In order to see that $\left(\begin{array}{l}* \\ *\end{array}\right) \Rightarrow(*)$ is a consequence of Esterle's theorem ([Es;4], p. 109, Th. 2.1), take any positive strictly decreasing sequence $\lambda_{0}, \lambda_{1}, \ldots$ satisfying (5). Then, by $\left(\begin{array}{l}* \\ *\end{array}\right)$,

$$
\sup _{(a, x) \in Z\left(\lambda_{n-1}\right)} \operatorname{dist}\left((a, x) ; Z\left(\lambda_{n}\right)\right) \leq 2 \gamma_{n} \lambda_{n-1}=2\left(\lambda_{n-1}-\lambda_{n}\right)
$$

for $n=1,2, \ldots$ Since $\sum_{n=1}^{\infty} 2\left(\lambda_{n-1}-\lambda\right)=2<\infty$, Esterle's theorem implies that $\bigcap_{n=0}^{\infty} \overline{Z\left(\lambda_{n}\right)} \neq \emptyset$, proving $(*)$.

Similar applications of the theorems of Pták and Esterle to the factorization problem are presented in $[\mathrm{K}-\mathrm{V}]$; $[\mathrm{P}-\mathrm{P}]$, pp. 134-137; [D-W], pp. 100104; [Es;4], pp. 115-119. In [P;3] a more sophisticated factorization theorem is deduced from the "induction theorem".

3.5. Extensions of the Cohen-Hewitt theorem to representations of some non-Banach algebras. It was observed in $[\mathrm{O}]$ and $[\mathrm{Cr}]$ that the argument of Koosis, presented in Remark 3, works in every Fréchet algebra $\left({ }^{7}\right)$ with a uniformly bounded left approximate identity (u.b.l.a.i.). This idea was taken up in [W.H.S], [M.K.S], [Vo;1] and [Vo;2]. Examples of Fréchet algebras with u.b.l.a.i. are discussed in $[\mathrm{O}]$ and $[\mathrm{Vo} ; 1]$. In [D] the u.b.l.a.i. is defined for any metrizable algebra, and the argument of Żelazko, described in our Sec. 3.3, is used for extending the factorization theorem to any essential continuous representation $T$ of a complete metrizable locally convex algebra $A$ with

$\left({ }^{7}\right)$ That is, an algebra which is a complete metrizable l.c.v.s. and, moreover, is locally multiplicatively convex. 
u.b.l.a.i. on a complete metrizable vector space $X$. In [AP] this last result is generalized to complete metrizable algebras which need not be locally convex, but are fundamental ([AP], p. 54, Def. 2.1).

3.6. Simultaneous factorization, factorization involving an analytic function, power factorization, and factorization by means of a one-parameter semigroup. The arguments discussed in Sections 2 and 3.1-4 were also used for proving theorems about simultaneous factorization of all the elements of a subset of $\overline{\operatorname{span}} T(A) X$ by means of a single element of $A$. For the case of Banach algebras see $[\mathrm{R} ; 2] ; \quad[\mathrm{V}] ; \quad[\mathrm{J}] ; \quad[\mathrm{T}] ; \quad[\mathrm{Ri}] ; \quad[\mathrm{S}-\mathrm{T}] ; \quad[\mathrm{C}-\mathrm{S}]$; [H-R;II], pp. 268-269, Theorem 32.23 and Corollary 32.24; [W.H.S]; [P1]; [B-D], p. 62, Corollary 12; [Ż], p. 23, Theorem 6.4; [Pal], pp. 538-539, Corollary 5.2.3. For extensions to Fréchet algebras (and more general algebras) see $[\mathrm{O}] ;$ [Cr]; [M.K.S]; [Z̈], p. 34, Theorem C.6.1; [Vo;1]; [Vo;2]; [D], Corollary 4.2 .

Cohen's basic idea of imbedding a Banach algebra $A$ into its unitization $A_{\mathrm{u}}$ and using an approximation in $A_{\mathrm{u}}$ also appears in [C-St] and $[\mathrm{S} ; 2]$ in connection with the factorization $y=T(f(a)) x$ where $f$ is an analytic function, in $[\mathrm{A}-\mathrm{S}],[\mathrm{Es} ; 1],[\mathrm{P} ; 2]$ and $[\mathrm{G}]$ where the power factorization is considered, and in $[\mathrm{S} ; 1,3,4]$ and [Pal], Sec. 5.3, pp. 543-548, in connection with the factorization by means of a one-parameter semigroup. The factorization theorem of A. M. Sinclair [S;4], pp. 35-37, concerns a representation $T$ of a complex Banach algebra $A$ on a complex Banach space $X$, and states that if there is a countable bounded two-sided approximate identity in $A$, then for every $y \in \overline{\operatorname{span}} T(A) X$ there are a holomorphic map $\mathbb{C} \ni z \mapsto x_{z} \in X$ and a holomorphic semigroup $\mathbb{C}^{+} \ni z \mapsto a^{z} \in A$ defined on the complex right half plane $\mathbb{C}^{+}=\{z \in \mathbb{C}: \operatorname{Re} z>0\}$, such that $y=x_{0}=T\left(a^{z}\right) x_{z}$ for every $z \in \mathbb{C}^{+}$, and $\lim _{U(\Psi) \ni z \rightarrow 0}\left\|a^{z} b-b\right\|_{A}=0$ for every $b \in A$ and every sector $U(\Psi)=\left\{z \in \mathbb{C}^{+}:|\operatorname{Arg} z| \leq \Psi\right\}, 0<\Psi<\pi / 2$. The growth properties of the holomorphic semigroups $\mathbb{C}^{+} \ni z \mapsto a^{z} \in A$ influence the structure of a Banach algebra $A$. See $[\mathrm{Es} ; 2,3],[\mathrm{S} ; 4],[\mathrm{W}],[\mathrm{G}-\mathrm{W}],[\mathrm{G}-\mathrm{R}]$ and other papers quoted there.

3.7. Factorization without a bounded approximate identity. Not all factorization theorems for topological algebras and their representations are proved by arguments of Cohen's type, related to bounded approximate identities. Indeed, the papers $[\mathrm{Pas}],[\mathrm{L}],[\mathrm{D}-\mathrm{M}],[\mathrm{P}-\mathrm{V}],[\mathrm{Vo} ; 1]$ and $[\mathrm{Ou}]$ deal with factorization and weak factorization in Banach algebras, and nonBanach function algebras (with pointwise multiplication) and convolution algebras, without bounded approximate identities. Also the proofs of weak factorization theorems for general separable Banach algebras, presented in [Pal], Sec. 5.3, pp. 549-552, have nothing in common with Cohen's argument. 


\section{References}

[A-S] G. R. Allan and A. M. Sinclair, Power factorization in Banach algebras with a bounded approximate identity, Studia Math. 56 (1976), 31-38.

[A;1] M. Altman, Factorisation dans les algèbres de Banach, C. R. Acad. Sci. Paris Sér. A 272 (1971), 1388-1389.

[A;2] -, Infinite products and factorization in Banach algebras, Boll. Un. Mat. Ital. 5 (1972), 217-229.

[A;3] -, Contracteurs dans les algèbres de Banach, C. R. Acad. Sci. Paris Sér. A 274 (1972), 399-400.

[A;4] -, Contractors, approximate identities and factorization in Banach algebras, Pacific J. Math. 48 (1973), 323-334.

$[\mathrm{A} ; 5] \quad-$, A generalization and the converse of Cohen's factorization theorem, Duke Math. J. 42 (1975), 105-110.

[AP] E. Ansari-Piri, A class of factorable topological algebras, Proc. Edinburgh Math. Soc. 33 (1990), 53-59.

[B-D] F. F. Bonsall and J. Duncan, Complete Normed Algebras, Springer, 1979.

[C] P. Cohen, Factorization in group algebras, Duke Math. J. 26 (1959), 199-205.

[C-S] H. S. Collins and W. H. Summers, Some applications of Hewitt's factorization theorem, Proc. Amer. Math. Soc. 21 (1969), 727-733.

[Cr] I. G. Craw, Factorization in Fréchet algebras, J. London Math. Soc. 44 (1969), 607-611.

[C-FT] P. C. Curtis, Jr. and A. Figà-Talamanca, Factorization theorems for Banach algebras, in: Function Algebras, F. T. Birtel (ed.), Scott, Foresman and Co., Chicago, IL, 1966, 169-185.

[C-St] P. C. Curtis, Jr. and H. Stetkaer, A factorization theorem for anatytic functions operating in a Banach algebra, Pacific J. Math. 37 (1971), 337-343.

[D-M] J. Dixmier et P. Malliavin, Factorisations de fonctions et de vecteurs indéfiniment différentiables, Bull. Sci. Math. (2) 102 (1978), 305-330.

[D] P. G. Dixon, Automatic continuity of positive functionals on topological involution algebras, Bull. Austral. Math. Soc. 23 (1981), 265-281.

[D-W] R. S. Doran and J. Wichmann, Approximate Identities and Factorization in Banach Modules, Lecture Notes in Math. 768, Springer, 1979.

[E;I] R. E. Edwards, Fourier Series, a Modern Introduction, Vol. I, 2nd ed., Springer, 1979.

[E;II] -, Fourier Series, a Modern Introduction, Vol. II, 2nd ed., Springer, 1982.

[Es;1] J. Esterle, Injection de semi-groupes divisibles dans des algèbres de convolution et construction d'homomorphismes discontinus de $\mathcal{C}(K)$, Proc. London Math. Soc. (3) 36 (1978), 59-85.

[Es;2] -, A complex-variable proof of the Wiener tauberian theorem, Ann. Inst. Fourier (Grenoble) 30 (1980), no. 2, 91-96.

[Es;3] J. Esterle, Elements for a classification of commutative radical Banach algebras, in: Proc. Conf. on Radical Banach Algebras and Automatic Continuity (Long Beach, 1981), J. M. Bachar et al. (eds.), Lecture Notes in Math. 975, Springer, Berlin, 1983, 4-65.

[Es;4] -, Mittag-Leffler method in the theory of Banach algebras and a new approach to Michael's problem, in: Banach Algebras and Several Complex Variables, F. Greenleaf and D. Gulick (eds.), Contemp. Math. 32, Amer. Math. Soc., 1984, 107-129. 
[F-L] H. G. Feichtinger and M. Leinert, Individual factorization in Banach modules, Colloq. Math. 51 (1987), 107-117.

[G-R] J. E. Galé and T. J. Ransford, On the growth of analytic semigroups along vertical lines, Studia Math. 138 (2000), 165-177.

[G-W] J. E. Galé and M. C. White, An analytic semigroup version of the BeurlingHelson theorem, Math. Z. 225 (1997), 151-165.

[G] N. Grønbæk, Power factorization in Banach modules over commutative radical Banach algebras, Math. Scand. 50 (1982), 123-134.

[G-L-R] S. L. Gulick, T. S. Liu and A. C. M. van Rooij, Group algebra modules. II, Canad. J. Math. 19 (1967), 151-173.

$[\mathrm{H}]$ E. Hewitt, The ranges of certain convolution operators, Math. Scand. 15 (1964), 147-155.

[H-R;II] E. Hewitt and K. A. Ross, Abstract Harmonic Analysis, Volume II, Structure and Analysis on Compact Groups, Analysis on Locally Compact Abelian Groups, 3rd printing, Springer, 1997.

[J] B. E. Johnson, Continuity of centralizers on Banach algebras, J. London Math. Soc. 41 (1966), 639-640.

[K] P. Koosis, Sur un théorème de Paul Cohen, C. R. Acad. Sci. Paris 259 (1964), 1380-1382.

[K-V] J. Křížková and P. Vrbová, A remark on a factorization theorem, Comment. Math. Univ. Carolin. 15 (1974), 611-614.

[L] M. Leinert, A commutative Banach algebra which factorizes but has no approximate units, Proc. Amer. Math. Soc. 55 (1976), 345-346.

[Ou] S. I. Ouzomgi, Factorization and automatic continuity for an algebra of infinitely differentiable functions, J. London Math. Soc. (2) 30 (1984), 265-280.

[O] J.-L. Ovaert, Factorisation dans les algèbres et modules de convolution, C. R. Acad. Sci. Paris Sér. A 265 (1967), 534-535.

[Pal] T. W. Palmer, Banach Algebras and the General Theory of *-Algebras, Volume I, Algebras and Banach Algebras, Encyclopedia Math. Appl. 49, Cambridge Univ. Press, 1994.

[Pas] W. L. Paschke, A factorable Banach algebra without bounded approximate unit, Pacific J. Math. 46 (1973), 249-251.

[P-V] H. Petzeltová and P. Vrbová, Factorization in the algebra of rapidly decreasing functions on $R_{n}$, Comment. Math. Univ. Carolin. 19 (1978), 489-499.

$[\mathrm{P}-\mathrm{P}] \quad$ F. A. Potra and V. Pták, Nondiscrete Induction and Iterative Processes, Res. Notes in Math. 103, Pitman, 1984.

[P;1] V. Pták, Un théorème de factorisation, C. R. Acad. Sci. Paris Sér. A 275 (1972), 1297-1299.

[P;2] -, Deux théorèmes de factorisation, ibid. 278 (1974), 1091-1094.

[P;3] —, Factorization in Banach algebras, Studia Math. 65 (1979), 279-285.

[Ri] M. Rieffel, On the continuity of certain intertwining operators, centralizers, and positive linear functionals, Proc. Amer. Math. Soc. 20 (1969), 455-457.

[R;1] W. Rudin, Factorization in the group algebra of the real line, Proc. Nat. Acad. Sci. U.S.A. 43 (1957), 339-340.

[R;2] - Representation of functions by convolutions, J. Math. Mech. 7 (1958), $103-115$

[S] R. Salem, Sur les transformations des séries de Fourier, Fund. Math. 33 (1939), 108-114.

[S-T] F. D. Sentilles and D. C. Taylor, Factorization in Banach algebras and the general strict topology, Trans. Amer. Math. Soc. 142 (1969), 141-152. 
[S;1] A. M. Sinclair, Bounded approximate identities, factorization, and a convolution algebra, J. Funct. Anal. 29 (1978), 308-318.

[S;2] - Cohen's factorization method using an algebra of analytic functions, Proc. London Math. Soc. 39 (1979), 451-468.

[S;3] —, Cohen elements in Banach algebras, Proc. Roy. Soc. Edinburgh Sect. A 84 (1979), 55-70.

[S;4] -, Continuous Semigroups in Banach Algebras, London Math. Soc. Lecture Note Ser. 63, Cambridge Univ. Press, 1982.

[M.K.S] M. K. Summers, Factorization in Fréchet modules, J. London Math. Soc. (2) 5 (1972), 243-248.

[W.H.S] W. H. Summers, Factorization in Fréchet spaces, Studia Math. 39 (1971), 209-216.

[T] D. C. Taylor, A characterization of Banach algebras with approximate unit, Bull. Amer. Math. Soc. 74 (1968), 761-766.

[V] N. T. Varopoulos, Sur les formes positives d'une algèbre de Banach, C. R. Acad. Sci. Paris Sér. A 258 (1964), 2465-2467.

[Vo;1] J. Voigt, Factorization in some Fréchet algebras of differentiable functions, Studia Math. 78 (1984), 333-347.

[Vo;2] - Factorization in Fréchet algebras, J. London Math. Soc. (2) 29 (1984), $147-152$.

[W] M. C. White, Strong Wedderburn decompositions of Banach algebras containing analytic semigroups, J. London Math. Soc. (2) 49 (1994), 331-342.

[Z;I] A. Zygmund, Trigonometric Series, Vol. I, 2nd ed., Cambridge Univ. Press, 1959.

[Ż] W. Żelazko, Banach Algebras, Elsevier, Amsterdam, and PWN-Polish Sci. Publ., Warszawa, 1973.

Faculty of Electrical Engineering

Technical University of Lublin

Nadbystrzycka 38A, P.O. Box 189

20-618 Lublin, Poland

E-mail: kisynski.wzipt@antenor.pol.lublin.pl

Reçu par la Rédaction le 28.6.2000

Révisé le 13.11.2000 